\title{
NUMERICALLY PREDICTED J-INTEGRAL AS A MEASURE OF CRACK DRIVING FORCE FOR STEELS 1.7147 AND 1.4762
}

\author{
Goran Vukelic \\ University of Rijeka, Faculty of Maritime Studies Rijeka, Croatia \\ e-mail: gvukelic@pfri.hr \\ JOSIP BRNIC \\ University of Rijeka, Faculty of Engineering, Rijeka, Croatia
}

\begin{abstract}
Fracture behavior of two types of steel (1.4762 and 1.7147) is compared based on their numerically obtained $J$-integral values. The $J$-integral are chosen to quantify the crack driving force using the finite element (FE) stress analysis applied to single-edge notched bend (SENB) and compact tensile (CT) type fracture specimens. The resulting $J$-values are plotted for growing crack length $(\Delta a$ - crack length extension) at different $a / W$ ratios $(a / W$ - relative crack length; $0.25,0.5,0.75)$. Slightly higher resulting values of the $J$-integral for 1.4762 than 1.7147 can be noticed. Also, higher $a / W$ ratios correspond to lower $J$-integral values of the materials and vice versa. $J$-integral values obtained by using the FE model of the CT specimen give somewhat conservative results when compared with those obtained by the FE model of the SENB specimen.
\end{abstract}

Keywords: crack, steel 1.7147, steel 1.4762, FE analysis

\section{Introduction}

Material imperfections and failures due to the manufacturing process coupled with severe service conditions can lead to flaw appearance in engineering structures. Consequently, crack occurrence and its growth can seriously affect integrity of such structures leading to catastrophic failure. In order to avoid such a scenario, proper selection of materials is a step of great importance in the process of structural design. Selection of an improper material may affect product profitability, reduce its service lifetime and finally result in appearance of flaws and failure. Several requirements have to be met during the material selection process. These requirements include adequate strength of the material, acceptable rigidity level, resistance to elevated temperatures, etc., but also the material must be sufficiently resistant to crack propagation.

The resistance of the material to crack propagation in fracture mechanics is usually described through one or more parameters obtained by experimental research, like crack tip opening displacement (CTOD), $J$-integral or stress intensity factor $K$. Of all the above mentioned, the $J$-integral is suitable for trying to quantify the material resistance to crack elongation when observing ductile fracture in metallic materials (Kossakowski, 2012). When dealing with a growing crack, the obtained $J$ values can be correlated to appropriate crack length extensions $\Delta a$ giving the resistance $R$ curve. Standardized experimental procedures are used to obtain the $R$ curve. Extensive experimental procedures can be, in some cases, accompanied or even substituted using some of the modern numerical methods, e.g. the finite element (FE) method. Recent research on the topic of numerical fracture mechanics includes accuracy check of $J$-integral values obtained by experiments, planar (2D) FE analysis, space (3D) FE analysis or the EPRI method (Qiao et al., 2014). FE analysis of Mode I fracture in a compact tensile (CT) specimen has been conducted to reveal effects on micro, meso and macroscale (Saxena and Ramakrishnan, 2007), 
while plastic geometry factors were determined numerically in order to calculate the $J$-integral from the load vs. crack mouth opening displacement or load-line displacement curve in the $J$ - $R$ curve test (Huang et al., 2014). Elastic and plastic constraint parameters for 3D problems were studied on single-edge notched bend (SENB) and CT specimens of non-standard configuration to characterize fracture resistance parameters (Shlyannikov et al., 2014). Research on explaining procedures that guarantee the domain independent property when calculating the $3 \mathrm{D} J$-integral for large deformation problems was carried out by Koshima and Okada (2015). A 3D domain integral method based on the extended FE method for extracting mixed-mode stress intensity factors was described by Wu et al. (2012).

The work presented in this paper is a comparison of numerically obtained $J$-integral values taken as a measure of the crack driving force for steels 1.7147 and 1.4762. Steel 1.7147 is usually used in production of spindles, pistons, bolts, levers, camshafts, gears, shafts, etc. The latter is a heat-resistant steel used in furnace industry, ceramics and cement industry, etc., i.e., in applications with high temperature and relatively low tensile requirements. Carbon, low alloy or high alloy ferritic steels can exhibit ductile fracture at elevated temperatures (Zhu and Joyce, 2015). Structures made of these or similar steels are more than susceptible to the flaw appearance and crack growth (Wagner et al., 2010; Zangeneh et al., 2014; Gojic et al., 2011). Observing these examples, it is easy to understand the need for fracture characterization of such materials.

\section{Material properties}

Two materials are compared: structural steel 1.7147 (AISI 5120, 20MnCr5) and high chromium stainless steel 1.4762 (AISI 446, X10CrAlSi25). Chemical compositions of the mentioned materials are given in Tables 1 and 2. Composition of steel 1.7147 can be compared to the standard EN 10084-2008. Here, the content of carbon equals the maximum standard value $(0.22 \%)$ while the rest of the alloying elements are within the prescribed values. Comparing steel 1.4762 to the standard EN 10095-1999, all of the alloying elements are in the standard ranges.

Table 1. Chemical composition of steel 1.7147 (wt\%) (Brnic et al., 2014a)

\begin{tabular}{|c|c|c|c|c|c|c|c|c|c|c|c|}
\hline Material & $\mathrm{C}$ & $\mathrm{Mn}$ & $\mathrm{Si}$ & $\mathrm{S}$ & $\mathrm{Nb}$ & $\mathrm{Cu}$ & $\mathrm{Cr}$ & $\mathrm{Ni}$ & $\mathrm{P}$ & $\mathrm{Ti}$ & Rest \\
\hline \hline 1.7147 & 0.22 & 1.23 & 0.29 & 0.025 & 0.03 & 0.06 & 1.11 & 0.08 & 0.021 & 0.02 & 96.914 \\
\hline
\end{tabular}

Table 2. Chemical composition of steel 1.4762 (wt\%) (Brnic et al., 2014b)

\begin{tabular}{|c|c|c|c|c|c|c|c|c|c|c|c|}
\hline Material & $\mathrm{C}$ & $\mathrm{Mn}$ & $\mathrm{Si}$ & $\mathrm{S}$ & $\mathrm{Mo}$ & $\mathrm{Al}$ & $\mathrm{Cr}$ & $\mathrm{Ni}$ & $\mathrm{P}$ & $\mathrm{V}$ & Rest \\
\hline \hline 1.4762 & 0.102 & 0.519 & 1.2 & 0.01 & 0.116 & 1.23 & 23.05 & 0.6855 & 0.0217 & 0.201 & 72.8648 \\
\hline
\end{tabular}

Engineering stress-strain $(\sigma-\varepsilon)$ diagrams for both steels are given in Fig. 1, while the yield strength $\sigma_{Y S}$, tensile strength $\sigma_{T S}$ and Young's modulus $E$ are given in Table 3.

Table 3. Yield strength $\sigma_{Y S}$, tensile strength $\sigma_{T S}$ and Young's modulus $E$ of the considered materials (Brnic et al., 2014a,b)

\begin{tabular}{|c|c|c|c|}
\hline Material & $\sigma_{Y S}[\mathrm{MPa}]$ & $\sigma_{T S}[\mathrm{MPa}]$ & $E[\mathrm{GPa}]$ \\
\hline \hline 1.7147 & 398 & 562 & 219 \\
\hline 1.4762 & 487 & 584 & 192 \\
\hline
\end{tabular}




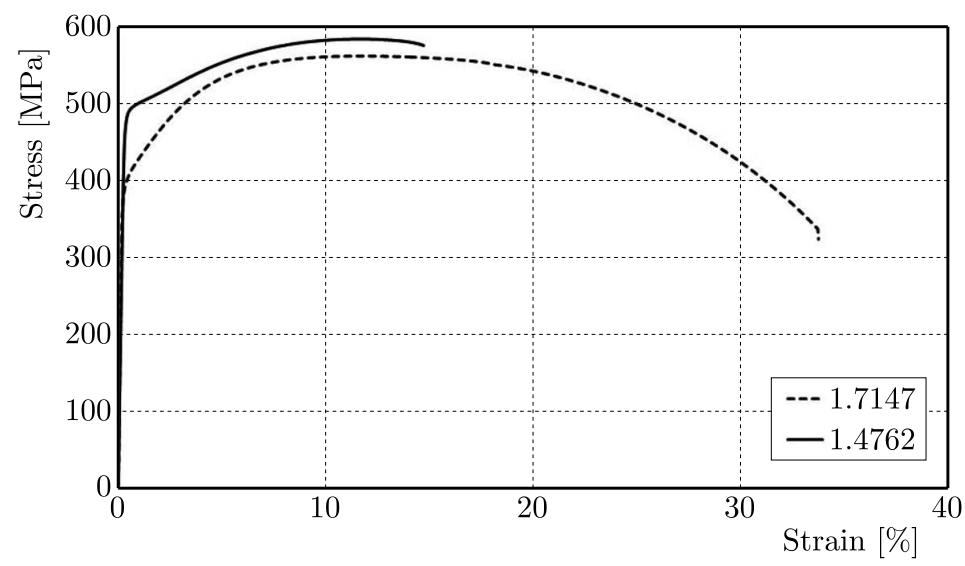

Fig. 1. Steel 1.7147 and steel 1.4762: uniaxial engineering stress-strain diagrams

\section{Importance of $J$-integral}

Rice (1968) introduced the $J$-integral as a path-independent integral that can be encircled around the tip of a crack and considered equally as an energy release rate parameter and a stress intensity parameter. In a $2 \mathrm{D}$ form and with reference to Fig. 2, it can be written as

$$
J=\int_{\Gamma}\left(w d y-T_{i} \frac{\partial u_{i}}{\partial x} d s\right)
$$

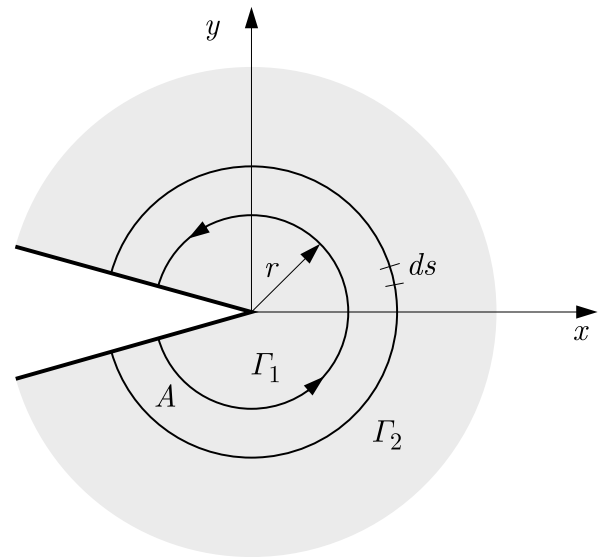

Fig. 2. J-integral arbitrary contour path enclosing the tip of a crack

Equation (3.1) comprises of $T_{i}=\sigma_{i j} n_{j}$ that are components of the traction vector, $u_{i}$ are the components of the displacement vector and ds is an incremental length along the integral contour $\Gamma$. The strain energy density $w$ can be written as

$$
w=\int \sigma_{i j} d \varepsilon_{i j}
$$

where $\varepsilon_{i j}$ is the sum of elastic and plastic strains at a specific point. The $J$-integral is path independent as long as the stress is a function of the strain alone and provided the crack tip is the only singularity within the contour. The $J$-integral equation shows that the energy of the integral contour increases for the crack growth per unit length. The $J_{I c}$ parameter, that can be 
derived, describes the fracture resistance of the material, i.e. required energy for crack growth per unit length when the contour $\Gamma$ must shrink to the crack tip

$$
J=\lim _{r \rightarrow 0} \int_{\Gamma}\left(w d y-T_{i} \frac{\partial u_{i}}{\partial x} d s\right)
$$

\section{Numerical prediction of $J$-integral}

The experimental single specimen test method following an elastic unloading compliance technique was numerically simulated in order to predict fracture behavior of steels 1.7147 and 1.4762 . It is an experimental test method that estimates the size of the expanding crack based on measured values of the crack mouth opening displacement. The resulting $J$ values serve as a fracture toughness parameter and can be correlated to crack extension values. The numerical procedure begins with FE stress analysis. Two-dimensional FE models of two types of fracture specimens, single edge notched bend (SENB) and compact tensile (CT), are defined according to the ASTM standard (2005), see Fig. 3. Three initial relative crack length $a / W(W=50 \mathrm{~mm})$ ratios are taken, $0.25,0.5$ and 0.75 . As for the material behavior, it is considered to be multilinear isotropic hardening. Specimens are discretized with 8-node isoparamateric quadrilateral elements. High deformation gradients occur in the yielding regions around the crack tip. That is why the FE mesh is refined there. Quasi-static load is imposed on the specimen in order to simulate the compliance procedure of the single specimen test method. Since the specimen is symmetrical, only half of it needs to be modelled. To simulate crack propagation, the node releasing technique has been used.

(a)

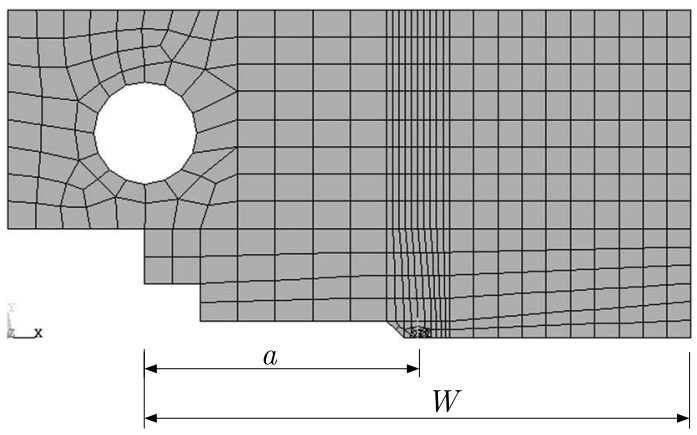

(b)

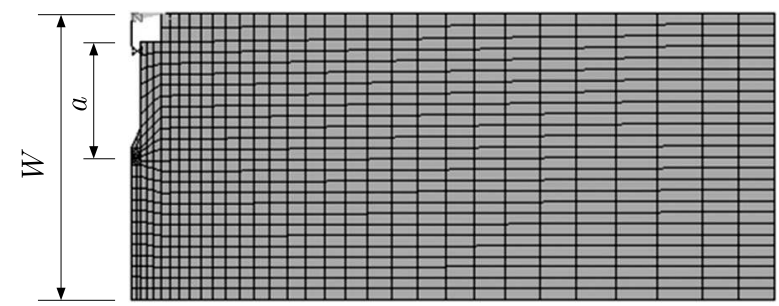

Fig. 3. Finite element model of: (a) CT specimen, (b) SENB specimen

The second step is to extract stress analysis results from the integration points of finite elements enclosing the crack tip. This results are used to evaluate $J$ values in the integration points by Eq. (4.1) (De Araujo et al., 2008) and sum them along the path $\Gamma$ that encloses the crack tip giving the total value of $J$, see Fig. 4

$$
J=\sum_{p=1}^{n p} G_{p} I_{p}\left(\xi_{p}, \eta_{p}\right)
$$

In Eq. (4.1), $G_{p}$ represents Gauss weighting factor, $n p$ stands for the number of integration points and $I_{p}$ is the integrand evaluated at each Gauss point $p$

$$
\begin{aligned}
I_{p} & =\left\{\frac{1}{2}\left[\sigma_{x x} \frac{\partial u_{x}}{\partial x}+\sigma_{x y}\left(\frac{\partial u_{x}}{\partial y}+\frac{\partial u_{y}}{\partial x}\right) \frac{\partial u_{x}}{\partial x}+\sigma_{y y} \frac{\partial u_{y}}{\partial y}\right] \frac{\partial y}{\partial \eta}\right. \\
& \left.-\left[\left(\sigma_{x x} n_{1}+\sigma_{x y} n_{2}\right) \frac{\partial u_{x}}{\partial x}+\left(\sigma_{x y} n_{1}+\sigma_{y y} n_{2}\right) \frac{\partial u_{y}}{\partial x}\right] \sqrt{\left(\frac{\partial x}{\partial \eta}\right)^{2}+\left(\frac{\partial y}{\partial \eta}\right)^{2}}\right\}_{g}
\end{aligned}
$$




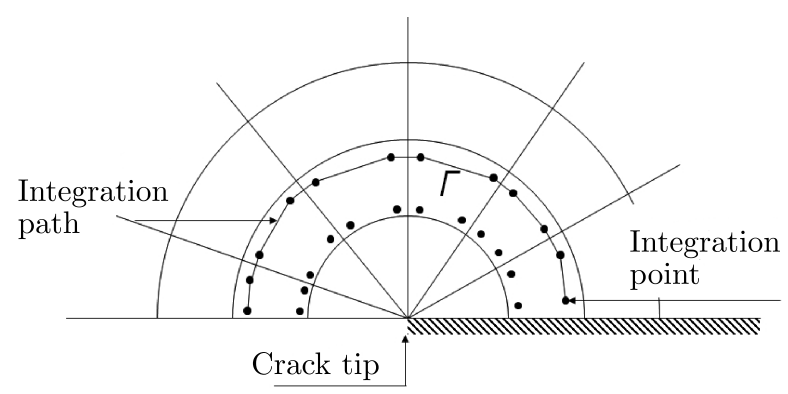

Fig. 4. J-integral path $\Gamma$ encircled around the crack tip through FE integration points

Although the crack tip plastic zone radius can be taken as variable using von Mises yield criterion (Bian, 2009), here it is taken as a constant value. Since a slight variation of $J$ values is possible in the numerical analysis, three different paths around the crack tip are defined in each example. The average value of these three paths is taken as the final value of the $J$-integral. In order to verify the procedure, the $J$-integral values are first compared to the available experimental results. Since there is no available experimental results for steels 1.7147 and 1.4762, the procedure has been first validated on steel 1.6310 (Narasaiah et al., 2010), Fig. 5 .

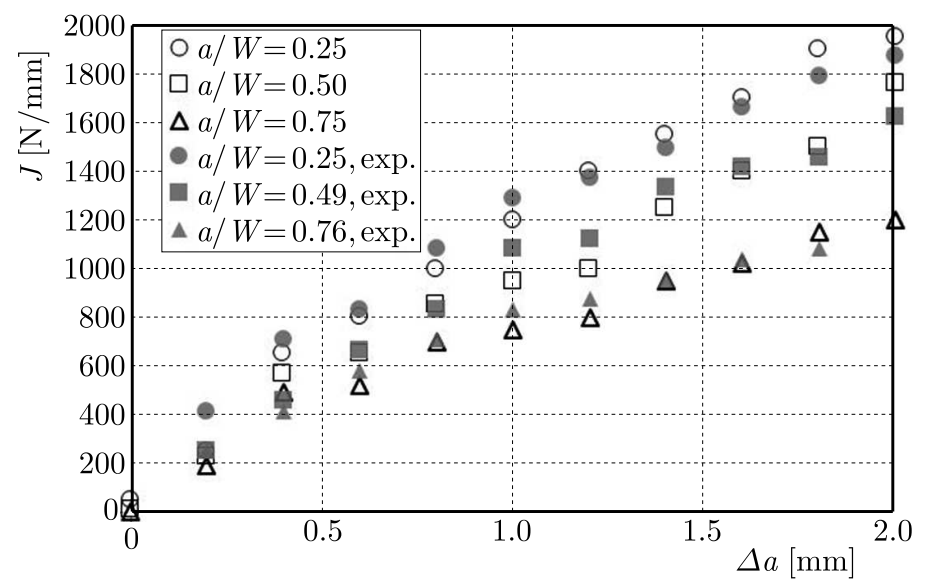

Fig. 5. Validation of numerically obtained $J$-integral values on steel 1.6310

Good compatibility of the experimental and numerical results encouraged further use of the numerical procedure for steels 1.7147 and 1.4762. Figures 6 and 7 show the final $J$ values for 1.7147 and 1.4762 taken as a measure of the crack driving force for different initial crack lengths $a / W$ according to the crack propagation $\Delta a$.
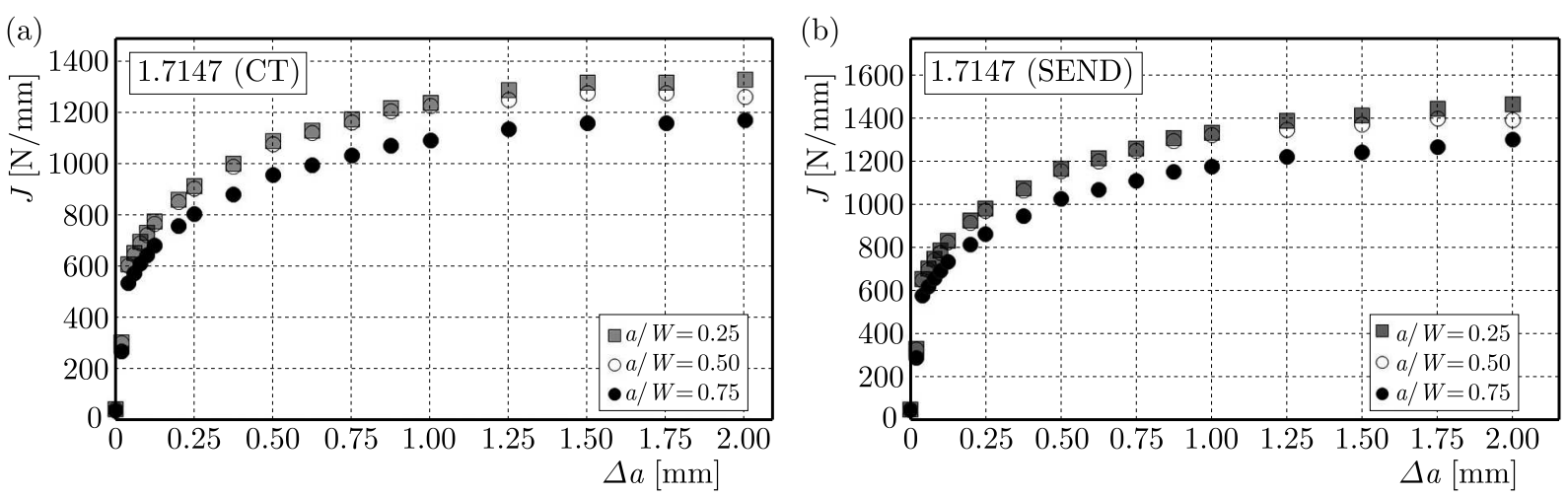

Fig. 6. J-integral values obtained numerically for steel 1.7147: (a) CT specimen, (b) SENB specimen 

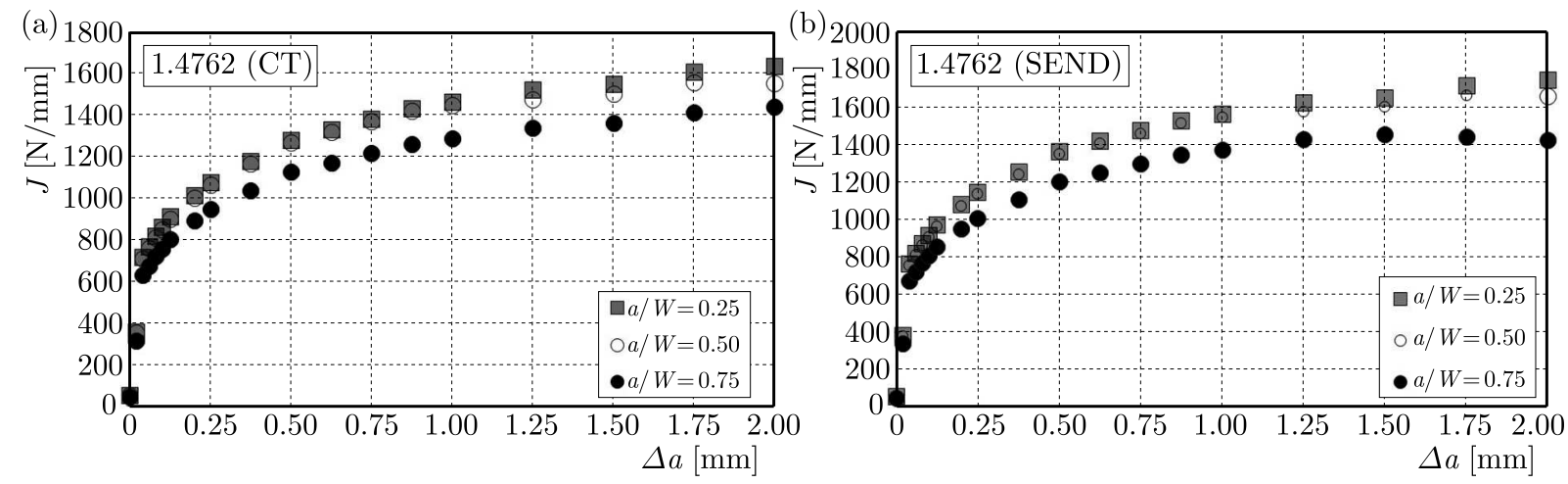

Fig. 7. J-integral values obtained numerically for steel 1.4762: (a) CT specimen, (b) SENB specimen

\section{Discussion}

Fracture behavior of steel 1.7147 and steel 1.4762 can be predicted based on the numerical investigation results presented in Figs. 6 and 7 using $J$-integral values as a measure of the crack driving force. Observing the obtained diagrams, it is clear that steel 1.4762 has slightly higher values of the $J$-integral than 1.7147 . This makes steel 1.4762 a bit more adequate to structures that need less susceptibility to fracture.

The predicted discrepancy in the numerically obtained $J$ values and, therefore, the difference in resistance to crack extension comparing steels 1.7147 and 1.4762 can be contributed to different composition and properties (Tables 1-3) of the two steels. Steel 1.4762 has a somewhat higher value of the nickel content which can add to the noted behavior. Nickel, as the alloying element, is usually added to stainless steels to reach a certain level of increased strength and hardness without compromising ductility and toughness levels. Nickel also improves the oxidization and corrosion resistance when added in suitable quantities to stainless steels. Although steel 1.7147 has an elevated chromium content (1.11\%) making it suitable for corrosive environment. Steel 1.4762 is a true stainless steel in which chromium exceeds $12 \%$ content (here $23.05 \%$ ) significantly improving corrosion resistance. Benefits of chromium as an alloying element in steel are also improved strength, hardenability, wear resistance and response to heat treatment.

Also, observing Figs. 6 and 7, lower $a / W$ ratios corresponding to higher $J$ values exhibit a trend observed by other authors (Cravero and Ruggieri, 2003). Also, the $J$-integral differs greatly for $a / W=0.75$ if matched with $a / W=0.25$ and 0.5 , then they tend to be close in values. In addition, $J$-integral values obtained by the $\mathrm{FE}$ model of the CT specimen give a bit conservative results when comparing them to those obtained from the SENB specimen. That can be ascribed to the specimen geometry and loading effect. As for the crack geometry, $a / W$ ratios are kept equal for both steels in relative specimens. That way, the influence of geometry on the difference in $J$ values for the two steels is negligible.

\section{Conclusion}

Numerical assessment of the $J$-integral for steels 1.7147 and 1.4762 can be useful as a prediction of the possible fracture behavior of materials. Although not validated by an experiment, the fine correspondence between numerical and experimental results for steel 1.6310 assures confidence in using $J$-integral values for steels 1.7147 and 1.4762 . In the structural design procedure that includes any of the considered material, the obtained results can be useful in the initial assessment of the material susceptibility to crack growth. 
The presented work intends to attract attention on the need for fracture behavior characterization of materials recommended for use in specific engineering components. Here, the novelty of the research lies in numerically predicted values of the $J$-integral taken as a measure of the crack driving force for steels 1.7147 and 1.4762 which are, according to the authors' knowledge, unavailable to construction designers in the presented form. Both materials offer improved corrosion resistance and can be considered for engineering applications intended to be used in corrosive environment and susceptible to crack growth and fracture like spiral bevel gears in truck differential systems prone to failure (Sekercioglu and Kovan, 2007) or stainless steel tubes found in recuperators and exposed to elevated temperatures that cause failures (Bhattacharyya et al., 2008). The results of the investigation presented in this paper can be used to avoid such failure scenarios.

\section{Acknowledgment}

Research presented in this paper has been supported by Croatian Science Foundation (project 6876) and by University of Rijeka (projects 13.09.1.1.01 and 13.07.2.2.04).

\section{References}

1. American Society for Testing and Materials (ASTM), 2005, Standard test method for measurement of fracture toughness E1820, ASTM, Baltimore

2. Bhattacharyya S., Das M.B., Sarkar S., 2008, Failure analysis of stainless steel tubes in a recuperator due to elevated temperature sulphur corrosion, Engineering Failure Analysis, 15, 6, $711-722$

3. BiAn L., 2009, Crack growth prediction and non-linear analysis for an elasto-plastic solid, International Journal of Engineering Science, 47, 3, 325-41

4. Brnic J., Turkalu G., Lanc D., Canadija M., Brcic M., Vukelic G., 2014a, Comparison of material properties: Steel 20MnCr5 and similar steels, Journal of Constructional Steel Research, 95, $81-89$

5. Brnic J., Turkalu G., Krscanski S., Lanc D., Canadija M., Brcic M., 2014b, Information relevant for the design of structure: ferritic-heat resistant high chromium steel X10CrAlSi25, Materials and Design, 63, 508-518

6. Cravero S., Ruggieri C., 2003, A two-parameter framework to describe effects of constraint loss on cleavage fracture and implications for failure assessments of cracked components, Journal of the Brazilian Society of Mechanical Sciences and Engineering, 25, 4, 403-412

7. De Araujo T.D., Roehl D., Martha L.F., 2008, An adaptive strategy for elastic-plastic analysis of structures with cracks, Journal of the Brazilian Society of Mechanical Sciences and Engineering, 30, 4, 341-350

8. Gojic M., Nagode A., Kosec B., Kozuh S., Savli S., Holjevac-Grguric T., Kosec L., 2011, Failure of steel pipes for hot air supply, Engineering Failure Analysis, 18, 8, 2330-2335

9. Huang Y., Zhou W., Yan Z., 2014, Evaluation of plastic geometry factors for SE(B) specimens based on three-dimensional finite element analyses, International Journal of Pressure Vessels and Piping, 123-124, 99-110

10. Koshima T., Okada H., 2015, Three-dimensional $J$-integral evaluation for finite strain elasticplastic solid using the quadratic tetrahedral finite element and automatic meshing methodology, Engineering Fracture Mechanics, 135, 34-63

11. Kossakowski P.G., 2012, Simulation of ductile fracture of S235JR steel using computational cells with microstructurally-based length scales, Journal of Theoretical and Applied Mechanics, 50, $589-607$ 
12. Narasaiah N., Tarafder S., Sivaprasad S., 2010, Effect of crack depth on fracture toughness of 20MnMoNi55 pressure vessel steel, Material Science and Engineering A, 527, 2408-2411

13. Qiao D., Changyu Z., Jian P., Xiaohua H., 2014, Experiment, Finite Element Analysis and EPRI Solution for $J$-integral of Commercially Pure Titanium, Rare Metal Materials Engineering, 42, 2, 257-263

14. RiCE J.R., 1968, A path independent integral and the approximate analysis of strain concentration by notches and cracks, Journal of Applied Mechanics, 35, 379-386

15. Saxena S., Ramakrishnan N., 2007, A comparison of micro, meso and macroscale FEM analysis of ductile fracture in a CT specimen (mode I), Computational Materials Science, 39, 1, 1-7

16. Sekercioglu T., Kovan V., 2007, Pitting failure of truck spiral bevel gear, Engineering Failure Analysis, 14, 4, 614-619

17. Shlyannikov V.N., Boychenko N.V., Tumanov A.V., Fernández-Canteli A., 2014, The elastic and plastic constraint parameters for three-dimensional problems, Engineering Fracture Mechanics, 127, 83-96

18. Wagner D., Ranc N., Bathias C., Paris P.C., 2010, Fatigue crack initiation detection by an infrared thermography method, Fatigue and Fracture of Engineering Materials and Structures, 33, $1,12-21$

19. Wu L., Zhang L., Guo Y., 2012, Extended finite element method for computation of mixed-mode stress intensity factors in three dimensions, Proceedia Engineering, 31, 373-380

20. Zangeneh S., Ketabchi M., Kalaki A., 2014, Fracture failure analysis of AISI 304L stainless steel shaft, Engineering Failure Analysis, 36, 155-165

21. Zhu X.K., Joyce J.A., 2015, Review of fracture toughness (G, K, J, CTOD, CTOA) testing and standardization, Engineering Fracture Mechanics, 85, 1-46 